\title{
ZERO-SET ULTRAFILTERS AND $\boldsymbol{G}_{\delta}$-CLOSURES IN UNIFORM SPACES
}

\author{
HOWARD CURZER and ANTHONY W. HAGER
}

(Received 20 July 1978)

Communicated by J. H. Rubinstein

\begin{abstract}
The paper examines the classes $\mathscr{X}_{1}$ and $\Gamma_{1}$ of Hausdorff uniform spaces which are $G_{\delta}$-closed in their Samuel compactifications, or completions. It is shown that the classes are epi-reflective, the reflections $k_{1}$ and $\gamma_{1}$ are described, $\mathscr{X}_{1}$ and $\Gamma_{1}$ are represented as epi-reflective hulls, membership in the classes is described by fixation of certain zero-set ultrafilters, and it is shown that $k_{1}=\gamma_{1}$ exactly on spaces without discrete sets of measurable power. The results include familiar facts about realcompact and topologically complete topological spaces and are closely connected with the theory of metric-fine uniform spaces.
\end{abstract}

Subject classification (Amer. Math. Soc. (MOS) 1970): primary 54 C 50, 54 E 15, 18 A 40; secondary 54 B 05, 54 B 10, 54 C 10, 54 C 30.

Keywords: uniform space, metric-fine space, zero-set, ultrafilter, $\boldsymbol{G}_{\delta}$-closure, realcompact, topologically complete, Katětov-Shirota, epi-reflective.

This paper examines the classes $\mathscr{K}_{1}$ and $\Gamma_{1}$ of Hausdorff uniform spaces which are $G_{\delta}$-closed in their Samuel compactifications, or completions. It is shown that the classes are epi-reflective, the reflections $k_{1}$ and $\gamma_{1}$ are described, $\mathscr{K}_{1}$ and $\Gamma_{1}$ are represented as epi-reflective hulls, membership in the classes is characterized by fixation of certain uniform $z$-ultrafilters, and it is shown that $k_{1}=\gamma_{1}$ exactly on spaces without discrete sets of measurable power.

For a Tychonoff space equipped with its finest compatible uniformity, membership in $\mathscr{K}_{1}$ is equivalent to realcompactness, and in $\Gamma_{1}$ to topological completeness (but uniform spaces not in $\mathscr{K}_{1}$ (or $\Gamma_{1}$ ) frequently have realcompact (or topologically complete) topology), so the results here include results from topology (for the most part, familiar ones). The analogy is quite faithful, as the results show. 
Rather, though, than from this analogy, the classes $\mathscr{K}_{1}$ and $\Gamma_{1}$ arose originally and naturally in the studies of metric-fine uniform spaces of Hager (1974) and M. D. Rice $(1975 \mathrm{a}, \mathrm{b})$. The connection is explained in Section 5, and we shall reprove here, more directly, some of the results from these papers.

\section{Epi-reflections and $G_{\delta}$-closures}

'Space' means Hausdorff uniform space, and spaces will be denoted $X, Y, \ldots$. Unif stands for the category with these objects, and maps are uniformly continuous functions. Essentially, we shall assume that the notion of an epi-reflective subcategory is a familiar one. For our purposes, Section 1 of Hager (1975) is a good introduction.

$\mathscr{K}$ is the subcategory of compact spaces. This is epi-reflective, and the reflection $k_{x}: X \rightarrow k X$ is the Samuel compactification. Each reflection morphism $k_{x}$ is a uniformly continuous homeomorphism with dense image (which is an embedding if and only if $X$ is precompact).

$\Gamma$ is the subcategory of complete spaces. This is epi-reflective, and the reflection $\gamma_{x}: X \rightarrow \gamma X$ is the completion. Each $\gamma_{x}$ is a dense embedding.

Now let $\mathscr{R}$ be an epi-reflective subcategory of Unif for which each reflection morphism $r_{x}$ is a homeomorphism (equivalently, $\mathscr{R} \supset \mathscr{K}$ )-such as $\mathscr{K}$ or $\Gamma$. Given such $\mathscr{R}$, let $\mathscr{R}_{1}$ be the class of spaces $X$ for which $r_{x}(X)$ (or less precisely, $X$ ) is $G_{\delta}$-closed in $r X$ (that is, if $p \in r X-r_{x}(X)$, then there is a $G_{\delta}$-set $G$ with $p \in G$ and $\left.G \cap r_{x}(X)=\varnothing\right)$.

1.1. Proposition. (a) $\mathscr{R}_{1}$ is productive and closed-hereditary, hence epi-reflective.

(b) The reflection $r_{1} X$ is, as a set, the $G_{\delta}$-closure of $X$ in $r X$, and the uniformity is the finest (that is, largest) one for which the relativization to $X$ is coarser than the uniformity of $X$.

Regarding the proof of 1.1 : the verification that $\mathscr{R}_{1}$ is productive and closedhereditary is routine; epi-reflectivity follows from Kennison (1965). For (b), the uniformity described is realized as the supremum of the uniformities whose relativizations to $X$ are coarser than $X$ 's; then the required universal mapping property is verified.

1.1(b) does not convey much feeling for what the uniformity of $r_{1} X$ really is, but we have not a more incisive description. The difficulties appear even for $k_{1} X$, as the following illustrates. (Ultimately, a good description of $k_{1} X$ will be obtained, but this is involved.) 
1.2. CoRollary. (a) If $r_{X}: X \rightarrow r X$ is an embedding, then $r_{1} X$ is a subspace of $r X$, and $X$ is a subspace of $r_{1} X$.

(b) For any $X, X \subset \gamma_{1} X \subset \gamma X$.

(c) If $X$ is precompact, then $X \subset k_{1} X \subset k X$.

1.3. Proposition. (a) For any $X, k_{1} X \cap \gamma X=\gamma_{1} X$ as sets.

(b) $K_{1} \subset \Gamma_{1}$.

Proof. It is well known that if $X$ is dense in $Y$, then $k X=k Y$ and $\gamma X=\gamma Y$. It follows that if $X$ is $G_{\delta}$-dense in $Y$ (that is, each nonvoid $G_{\delta}$-set in $Y$ meets $X$ ), then $k_{1} X=k_{1} Y$ and $\gamma_{1} X=\gamma_{1} Y$. From this, (a) follows, and (b) follows from (a).

\section{Closed subspaces of products}

This section concerns representing $\mathscr{K}_{1}$ and $\Gamma_{1}$ as epi-reflective hulls or something similar.

The epi-reflective hull $\mathscr{R}(\mathscr{A})$ of the class $\mathscr{A}$ of spaces consists of spaces isomorphic to a closed subspace of a product of members of $\mathscr{A}$. For example: $\mathscr{K}=\mathscr{R}[0,1]$ (suppressing some parentheses); with $\mathscr{M}$ the class of metric spaces and $\mathscr{H}$ the complete metric spaces, $\mathscr{R}(\mathscr{K})=\Gamma$.

In what follows, $(0,1]$ carries its usual uniformity: $\gamma(0,1]=k(0,1]=[0,1]$.

2.1. Proposition. (a) (Rice (1975b)) $\Gamma_{1}=\mathscr{R}(\mathscr{M})$.

(b) These conditions on $X$ are equivalent:

(1) $X \in \mathscr{K}_{1}$.

(2) The evaluation $e: X \rightarrow \Pi\left\{(0,1]_{f}: f \in U(X,(0,1])\right\}$ has closed range.

(3) There is $Y \in \mathscr{R}(0,1]$ and a perfect map $f: X \rightarrow Y$.

(c) $\mathscr{K}_{1} \cap$ (Precompact spaces) $=\mathscr{R}(0,1]$.

We shall prove 2.1 by the technique of 'generalized perfect maps' developed in Hager (1975): For epi-reflective $\mathscr{R} \supset \mathscr{K}$, a (uniformly continuous) map $f: X \rightarrow Y$ is called $\mathscr{R}$-perfect if $r f\left(r X-r_{x}(X)\right) \subset r Y-r_{y}(Y)$. Given a class $\mathscr{A}$ of spaces, the class of all $X$ for which there are $A \in \mathscr{A}$ and $\mathscr{R}$-perfect $f: X \rightarrow A$ is denoted $p_{\mathscr{R}} \mathscr{\mathscr { A }}$ (and called the $\mathscr{R}$-perfect hull). A $\mathscr{K}$-perfect map is called just 'perfect', and $p_{\mathscr{X}} \mathscr{A}$ is familiar as the "left-fitting hull'.

2.2. LeMMA. Let $\mathscr{R}$ be epi-reflective with $\mathscr{R} \supset \mathscr{K}$, and suppose that $r(0,1]=[0,1]$. Then these conditions on $X$ are equivalent:

(a) $X \in \mathscr{R}_{1}$.

(b) For each $p \in r X-r_{x}(X)$, there is $g \in U(r X,[0,1])$ with $g(p)=0$ and $g\left(r_{x}(X)\right) \subset(0,1]$. 
(c) For each $p \in r X-r_{x}(X)$, there is $f \in U(X,(0,1])$ with $r f(p)=0$.

(d) The evaluation $e: X \rightarrow \prod\left\{(0,1]_{f} \mid f \in U(X,(0,1])\right\}$ is $\mathscr{R}$-perfect.

(e) $X \in p_{\mathscr{R}} \mathscr{R}(0,1]$.

PROOF. Sketch: (a) $\Rightarrow$ (b). If $G$ is a $G_{\delta}$ and $p \in G$, there is a zero-set $Z(g)$ with $p \in Z(g) \subset G$.

(b) $\Rightarrow$ (c). Since $r(0,1]=[0,1]$, a $g$ as in (b) is an $r f$ as in (c).

(c) $\Rightarrow$ (d). 2.10 of Hager (1975).

(d) $\Rightarrow$ (e). Obvious.

(e) $\Rightarrow(a)$. If $f: X \rightarrow Y$ is $\mathscr{R}$-perfect, then whenever $G$ is a $G_{\delta}$ in $r Y$ missing $r_{y}(Y)$, $f^{-1}(G)$ is a $G_{\delta}$ in $r X$ missing $r_{x}(X)$.

Proof of 2.1. (b) We take $\mathscr{R}=\mathscr{K}$ in 2.3. Then, (1) $\Rightarrow$ (2) by $2.3((\mathrm{a}) \Leftrightarrow(\mathrm{e}))$. Assuming (1), 2.3(d) holds. But a $\mathscr{K}$-perfect map has closed range (5.2 of Hager (1975)), so (2) holds. If (2) holds, then $e$ is $\mathscr{K}$-perfect because $e$ is a homeomorphism (2.6 of Hager (1975)).

(c) From (b) and the fact that $X$ is precompact if and only if $e$ is an embedding.

(d) We shall show that $\mathscr{R}(\mathscr{M}) \subset \Gamma_{1} \subset p_{\Gamma} \mathscr{R}(\mathscr{M}) \subset \mathscr{R}(\mathscr{M})$. First, if $M \in \mathscr{M}$, then $\gamma M$ is metric with points $G_{\delta}$-sets. Thus, $\mathscr{H} \subset \Gamma_{1}$; so $\mathscr{R}(\mathscr{M}) \subset \Gamma_{1}$ by $1.2($ a). Next, we have $\Gamma_{1}=p_{\Gamma} \mathscr{R}(0,1] \subset p_{\Gamma} \mathscr{R}(\mathscr{M})$ (by $2.2 ;$ then $\left.(0,1] \in \mathscr{M}\right)$. Finally, if $X \in p_{\Gamma} \mathscr{R}(\mathscr{M})$, then there are $A \in \Gamma, B \in \mathscr{R}(\mathscr{H})$, and a closed embedding of $X$ in $A \times B$, by 4.4 of Hager (1975). Since $\Gamma=\mathscr{R}(\mathscr{M}) \subset \mathscr{R}(\mathscr{M})$, we have $A \times B \in \mathscr{R}(\mathscr{M})$, hence $X \in \mathscr{R}(\mathscr{H})$.

2.3. Remark. 2.1(b), read as a statement in Tychonoff spaces, is true, but the more familiar version has the real line replacing $(0,1]$ in (2) and (3). In Unif, this replacement is not valid. Let $X$ be the metric hedgehog with $\aleph_{0}$ spines. $X$ is not precompact, but each $f \in U(X, R)$ is bounded. Then the evaluation

$$
e: X \rightarrow \prod\left\{R_{f} \mid f \in U(X, R)\right\}
$$

has precompact range which, if closed, would be compact; then $X$ would be compact. Likewise, if there were perfect $f: X \rightarrow Y \in \mathscr{R}(R)$, again $Y$ would be compact, and $X$ also.

\section{Zero-set ultrafilters}

For $f$ a real-valued function, $Z(f) \equiv\{x \mid f(x)=0\}$. For $X \in$ Unif, a (uniform) zero-set of $X$ is a member of $\mathscr{Z}(X) \equiv\{Z(f) \mid f$ bounded in $U(X, R)\}$. A $z$-ultrafilter on $X$ is a maximal filter in $\mathscr{Z}(X)$. 
A $z$-ultrafilter $\mathscr{F}$ has the countable intersection property (cIP), if $(\mathscr{F} \subset \mathscr{F}$, $\mathscr{F}_{0}$ countable $\Rightarrow \bigcap \mathscr{F}_{0} \neq \varnothing$ ). It is easy to see that if $\mathscr{F}$ has cIP, then $\mathscr{F}$ is closed under countable intersection (because $\mathscr{Z}(X)$ is).

A family $\mathscr{D}$ of subsets of $X$ is called discrete if there is a uniformly continuous pseudometric $\rho$ and $\varepsilon>0$ such that $\rho(A, B) \geqslant \varepsilon$ for different $A, B \in \mathscr{D}$. If $\mathscr{D}_{1}, \mathscr{D}_{2}, \ldots$ are discrete, then $\bigcup \mathscr{D}_{n}$ is called $\sigma$-discrete.

If $\mathscr{B}$ is a family of subsets of $X$, then $\operatorname{co} \mathscr{B} \equiv\{X-B \mid B \in \mathscr{B}\}$. A $z$-ultrafilter $\mathscr{F}$ is said to have the $\operatorname{co}(\sigma$-discrete $)$ intersection property, or $\operatorname{co}(\sigma$-disc) IP, if $(\mathscr{F} \subset \mathscr{F}$, $\operatorname{co} \mathscr{F}_{0} \sigma$-discrete $\left.\Rightarrow \bigcap \mathscr{F} \neq \not \varnothing\right)$. Equivalently, if $(\mathscr{D} \subset \operatorname{co} \mathscr{F}, \mathscr{D} \sigma$-discrete $\Rightarrow \bigcup \mathscr{D} \neq X)$. Evidently, this property entails cIP. (See 3.3 regarding closure under such intersections.)

For $p \in k X$, let $\mathscr{F}_{p}=\{Z(f) \mid k f(p)=0, f$ bounded in $U(X, R)\}$. (Note that $p \notin k_{1} X$ if and only if $\emptyset \in \mathscr{F}_{p}$; so $\mathscr{F}_{p}$ is not always a filter.) A $z$-ultrafilter is called fixed if $\mathscr{F}=\mathscr{F}_{p}$ for some $p \in X$.

3.1. Proposition. Let $\mathscr{F}$ be a z-ultrafilter on $X$.

(a) $\mathscr{F}$ has cIP if and only if there is (unique) $p \in k_{1} X$ with $\mathscr{F}=\mathscr{F}_{p}$.

(b) These are equivalent:

(1) $\mathscr{F}$ has co(o-disc) IP;

(2) $\mathscr{F}$ has cIP and is Cauchy;

(3) there is (unique, $p \in \gamma_{1} X$ with $\mathscr{F}=\mathscr{F}_{p}$.

3.2. CoRollary. $X \in \mathscr{K}_{1}$ (resp., $\Gamma_{1}$ ) if and only if each z-ultrafilter with cIP (resp., co( $\sigma$-disc IP), is fixed.

Prior to the proof, a few remarks are in order. A topological zero-set (that is, of a continuous function) need not be a uniform zero-set. Let $X$ be an uncountable set with the uniformity inherited from the one-point compactification of discrete $X$. Then $\mathscr{Z}(X)$ consists of finite and co-countable sets, but, topologically, every subset is zero. (Note that Chapter 15 of Gillman and Jerison (1960) speaks of topological zero sets.)

In Tychonoff spaces, the compact reflection $(\beta)$ is the space of all topological $z$-ultrafilters, but not so in Unif. For $X \in$ Unif, the space of all $z$-ultrafilters is a generally much larger 'compactification' than $k X$, called $H\left(A^{*}(k X)\right)$ and pretty thoroughly analysed in Hager (1969); 3.1(a) above more or less follows from 4.2 and 5.3 there. (The space is also called an 'Alexandroff compactification'; see 7.5 and Section 9 of Hager (1974).)

Proof of 3.1. (a) Let $p \in k_{1} X$. We show that $\mathscr{F}_{p}$ is a $z$-ultrafilter with cIP. First, $\mathscr{F}_{p}$ is a $z$-filter: If $Z(f)$ and $Z(g) \in \mathscr{F}_{p}$, then $Z(f) \cap Z(g)=\ldots=Z(|k f|+|k g|) \cap X$, which is nonvoid because $p \in k_{1} X$; If $Z(g) \supset Z(f) \in \mathscr{F}_{p}$, we can arrange it that 
$0 \leqslant g \leqslant f$, hence $0 \leqslant k g \leqslant k f$, so $k g(p)=0$ and $Z(g) \in \mathscr{F}_{p}$. Next, $\mathscr{F}_{p}$ is maximal: If $Z(f) \notin \mathscr{F}_{p}$, then $p \notin Z(k f)$ and there is (by complete regularity of $\left.k X\right) Z(k g)$ containing $p$ and missing $Z(k f)$; then $Z(g) \in \mathscr{F}_{p}$, while $Z(f) \cap Z(g)=\varnothing$. Finally, $\mathscr{F}_{p}$ has cIP. Let $Z\left(f_{1}\right), Z\left(f_{2}\right), \ldots \in \mathscr{F}_{p}$, and suppose (as we may) that $0 \leqslant f_{n} \leqslant 2^{-n}$; now $p \in$ each $Z\left(k f_{n}\right)$, hence $p \in \bigcap_{n} Z\left(k f_{n}\right)=Z\left(\sum k f_{n}\right)=Z\left(k \sum f_{n}\right)$; so

$$
\cap Z\left(f_{n}\right)=X \cap Z\left(k \sum f_{n}\right)=Z\left(\sum f_{n}\right) \in \mathscr{F}_{p} .
$$

Conversely, suppose $\mathscr{F}$ has cIP. Then there is $p \in \bigcap\{Z \mid Z \in \mathscr{F}\}$ (closures in $k X$ ), because the family has the finite IP and $k X$ is compact. Let $G$ be a $G_{\delta}$ containing $p$. We may suppose that $G=\bigcap_{n} Z_{n}$, where each $Z_{n}$ is a zero-set of $k X$ with $p$ in its interior. Clearly then, $Z_{n} \cap Z \neq \varnothing$ for each $Z \in \mathscr{F}$. Thus each $Z_{n} \cap X \in \mathscr{F}$ (since $\mathscr{F}$ is maximal), and therefore $G \cap X=\bigcap_{n}\left(Z_{n} \cap X\right) \neq \varnothing$, by cIP. So $p \in k_{1} X$. Thus, by the other half of this proof above, $\mathscr{F}_{p}$ is a $z$-ultrafilter. Since clearly, $\mathscr{F} \subset \mathscr{F}_{p}$, equality follows.

(b) (1) $\Rightarrow(2)$. Suppose $\mathscr{F}$ has co( $\sigma$-disc) IP, and let $\mathscr{U}$ be a uniform cover of $X$. Choose a metric space $M$ and uniformly continuous $f: X \rightarrow M$ such that $f^{-1}(\mathscr{S})<\mathscr{U}$, where $\mathscr{S}$ is the collection of 1 -spheres in $M$ (see Isbell (1964)). Now choose a $\sigma$-discrete open cover $\mathscr{V}$ refining $\mathscr{S}$, by Stone (1948). It suffices to show that some member of $\mathscr{F}$ is contained in a member of $f^{-1}(\mathscr{V})$. If not, then given $V \in \mathscr{V}$, $f^{-1}(V) \ngtr F$ for each $F \in \mathscr{F}$; that is, $\left(X-f^{-1}(V)\right) \cap F \neq \varnothing$. In $M$, each closed set is a zero-set, so $X-f^{-1}(V) \in \mathscr{F}$. But $\bigcap_{V}\left(X-f^{-1}(V)\right)=\varnothing$, a contradiction.

(2) $\Rightarrow$ (3). Let $\mathscr{F}$ be Cauchy, with CIP. Then $\mathscr{F}=\mathscr{F}_{p}$ for $p \in k_{1} X$, by (a). If $\mathscr{F} *$ is the filter in the power set of $\gamma X$ generated by $\mathscr{F}$, then $\mathscr{F} *$ is Cauchy on $\gamma X$, and converges to some $q \in \gamma X$. If $q \neq p$, then choose a zero-set neighbourhood $Z_{q}$ of $q$ in $k X$ not containing $p$, then a zero-set $Z_{p}$ of $k X$ containing $p$ and missing $Z_{q}$. Then $Z_{p} \cap X$ and $Z_{q} \cap X$ are disjoint members of $\mathscr{F}_{p}$, a contradiction. Thus $p=q$, and $p \in \gamma X \cap k_{1} X=\gamma_{1} X$ (using 1.3).

(3) $\Rightarrow$ (1). Let $p \in \gamma_{1} X$ and let $\mathscr{D} \subset \operatorname{co} \mathscr{F}$ be $\sigma$-discrete. So $\mathscr{D}=\bigcup \mathscr{D}_{n}$, where for each $n$ there is a uniformly continuous pseudometric $\rho_{n}$ and $\varepsilon_{n}>0$ such that $\mathscr{D}_{n}$ is $\varepsilon_{n}$-discrete for $\rho_{n}$. Each $\rho_{n}$ has an extension to a uniformly continuous pseudometric $\gamma \rho_{n}$ on $\gamma X$. Let $Z_{n}=\left\{x \in X \mid \gamma \rho_{n}(p, x)=0\right\}$. Clearly, $Z_{n} \in \mathscr{F}_{p}$. Then $Z \equiv \bigcap_{n} Z_{n} \in \mathscr{F}_{p}$ as well.

Now suppose $X=\bigcup\left\{D \mid D \in \bigcup \mathscr{D}_{n}\right\}$. Set $X_{n}=\bigcup\left\{D \mid D \in \mathscr{D}_{n}\right\}$, so $X=\bigcup X_{n}$. Fixing $n$, if $Z \cap X_{n} \neq \varnothing$ then there is a single $D_{0}^{n} \in \mathscr{D}_{n}$ with $Z \cap X_{n} \subset D_{0}^{n}$. (For, choose $p_{0} \in Z \cap X_{n}$, then unique $D_{0}^{n} \in \mathscr{D}_{n}$ with $p_{0} \in D_{0}^{n}$. Then $\rho_{n}\left(p_{0}, D\right) \geqslant \varepsilon_{n}>0$ for any $D \in \mathscr{D}_{n}$ with $D \neq D_{0}^{n}$. Thus $\varnothing=D \cap Z_{n} \supset D \cap Z$.) Thus, $A=\bigcup_{n}\left(Z \cap X_{n}\right) \subset \bigcup_{n} D_{0}^{n}$. But $\bigcup_{n} D_{0}^{n} \in \operatorname{co} \mathscr{F}_{p}$ so $X-\bigcup_{n} D_{0}^{n}$ and $Z$ are disjoint members of $\mathscr{F}_{p}$, a contradiction.

It is frequently convenient to know that a $z$-ultrafilter with cIP is closed under countable intersection (as in the proof of 3.1), and so one naturally wonders about closure conditions for $z$-ultrafilters with $\operatorname{co}(\sigma$-disc $)$ IP. The difficulty is that a 
union of a discrete family of uniform cozero sets need not be cozero. Indeed, if $X$ has such a discrete family $\mathscr{D}$, then for any $p \in X-\mathrm{U} \mathscr{D}, \mathscr{F}_{p}$ has co( $\sigma$-disc) IP of course, $\mathscr{D} \subset \operatorname{co} \mathscr{F}_{p}$ but $\bigcup \mathscr{D} \notin \operatorname{co} \mathscr{F}_{p}$. However:

\subsection{PROPOSITION. In a uniform space $X$}

(a) These properties of a discrete family $\mathscr{D}$ are equivalent.

(1) $\cup \mathscr{D}$ is cozero.

(2) For each $\mathscr{D}^{\prime} \subset \mathscr{D}, \cup \mathscr{D}^{\prime}$ is cozero.

(3) $\mathscr{D}=\{\operatorname{cozg} \mid g \in \mathscr{P}\}$ for some equiuniformly continuous family $\mathscr{S} \subset U(X, R)$.

(b) A z-ultrafilter $\mathscr{F}$ has co( $\sigma$-disc) IP if and only if $\mathscr{F}$ has cIP, and $\bigcup \mathscr{D} \in \operatorname{coF}$ whenever $\mathscr{D}$ is as in (a).

Proof. (a) (2) $\Rightarrow(1)$ is obvious. (1) $\Rightarrow$ (3). Let $\mathscr{D}$ be $\varepsilon$-discrete for $\rho$, let $\bigcup \mathscr{D}=\operatorname{coz} f$ with $0 \leqslant f \leqslant 1$, and for $D \in \mathscr{D}$, let $f_{D}=\left((\varepsilon-\rho(0, D)) \vee 2 \varepsilon\right.$. Then $\mathscr{S}=\left\{f_{0} \mid D \varepsilon \mathscr{D}\right\}$ works. (3) $\Rightarrow$ (2). For $\mathscr{D}^{\prime}=\left\{\operatorname{coz} g \mid g \in \mathscr{S}^{\prime}\right\}, \cup \mathscr{D}^{\prime}=\operatorname{coz} \sum\left\{g \mid g \in \mathscr{S}^{\prime}\right\}$.

(b) If $\mathscr{F}$ has co( $\sigma$-disc) IP, then $\mathscr{F}$ has cIP of course. Suppose $\mathscr{D}$ is discrete, $\mathscr{D} \subset \operatorname{co} \mathscr{F}, \bigcup \mathscr{D}$ is cozero, but $\bigcup \mathscr{D} \notin \operatorname{co} \mathscr{F}$. Then $Z=X-\bigcup \mathscr{D} \notin \mathscr{F}$, so there is $Z_{0} \in \mathscr{F}$. with $Z \cap Z_{0}=\varnothing$. Then $\left\{\left\{X-Z_{0}\right\}\right\} \cup \mathscr{D} \subset \operatorname{co} \mathscr{F}$ is $\sigma$-discrete, but

$$
\left(X-Z_{\mathbf{0}}\right) \cup \cup \mathscr{D}=X \text {. }
$$

Conversely, if $\mathscr{F}$ has the stated properties, then condition (2) in 3.1 can be derived exactly as $(1) \Rightarrow(2)$ there.

\section{4. $\mathscr{K}_{1}$ versus $\Gamma_{1}$}

The main result here is a uniform version of the Katétov-Shirota Theorem. It is almost exactly analogous to the (topological) version presented in Curzer and Hager (1976); so we can be brief.

Recall that a set $S$ has measurable power if there is a countably additive $\{0,1\}$-valued measure $\mu$ on the power set of $S$, with $\mu(\{p\})=0$ for each $p \in S$ and $\mu(S)=1$; equivalently, if each ultrafilter in the power set with cIP is fixed (12.2 of Gillman and Jerison (1960)).

Let $\mathcal{N}$ be the class of spaces which have no discrete set of measurable power; equivalently, each uniform cover has a uniform refinement of nonmeasurable power. It is not hard to see that $\mathscr{N}$ is productive and hereditary, hence epi-reflective in Unif; let $n:$ Unif $\rightarrow \mathscr{N}$ denote the reflecting functor. It can be shown that a base for $n X$ is the set of all uniform coverings of $X$ of nonmeasurable power. (See Isbell (1964), p. 52.) 
4.1. Proposition. $\mathscr{K}_{1}=\Gamma_{1} \cap \mathscr{N}$ and $k_{1}=\gamma_{1} \circ n$.

4.2. LEMMA. For any $X$, if $\mathscr{F}$ is a $z$-ultrafilter with $c I P$, then whenever $\mathscr{D} \subset \operatorname{co} \mathscr{F}$, $\mathscr{D}$ is discrete of nonmeasurable power, and $\bigcup \mathscr{D}^{\prime}$ is cozero for each $\mathscr{D}^{\prime} \subset \mathscr{D}$, then $\bigcup \mathscr{D} \in \operatorname{co} \mathscr{F}$.

Hence, if $X \in \mathscr{N}$, then each $z$-ultrafilter with cIP has co( $\sigma$-disc $) I P$.

Proof. For the first part, the proof of 7 of Curzer and Hager (1976) can be copied. (The reference is to the corresponding statement for Tychonoff spaces. There, the hypothesis that the sets $\bigcup \mathscr{S}^{\prime}$ be cozero is unnecessary.)

The second part follows using 3.3.

ProOF of 4.1. Let $X \in \Gamma_{1} \cap \mathscr{N}$, and let $\mathscr{F}$ be a $z$-ultrafilter with cIP. By 4.2, $\mathscr{F}$ has $\operatorname{co}\left(\sigma\right.$-disc) IP. Since $X \in \Gamma_{1}, \mathscr{F}$ is fixed, and thus $X \in \mathscr{K}_{1}$ (using 3.2 twice).

Now, $\mathscr{K}_{1} \subset \Gamma_{1}$, by 1.3. Let $X \in \mathscr{K}_{1}$ and let $D$ be discrete in $X$. Then $D$ is closed, so by $1.1 D \in \mathscr{K}_{1}$. The relative uniformity on $D$ is discrete, so $\mathscr{Z}(D)$ is the power set $\mathscr{P}(\mathrm{D})$. So saying that $D \in \mathscr{K}_{1}$ is saying that $D$ has nonmeasurable power. Thus $\mathscr{K}_{1} \subset \mathscr{N}$.

We show that $k_{1}=\gamma_{1} \circ n$. Given $X, \gamma_{1} n X \in \Gamma_{1}$ since $\gamma_{1}$ operates last. It is trivial to check that $\gamma \mathscr{N} \subset \mathscr{N}$; thus $\gamma n X \in \mathscr{N}$. Since $\mathscr{N}$ is hereditary and $\gamma_{1} n X \subset \gamma n X$ (1.2), $\gamma_{1} n X \in \mathscr{N}$ as well. Thus $\gamma_{1} n X \in \mathscr{K}_{1}$. Now let $Y \in \mathscr{K}_{1}$, and let $f \in U(X, Y)$. Then $n f \in U(n X, Y)$ (since $Y \in \mathscr{N})$, and $\gamma_{1} n f \in U\left(\gamma_{1} n X, Y\right)$ (since $\left.Y \in \Gamma_{1}\right)$. Uniqueness of $\gamma_{1} n f$ (as a 'lift' of $f$ over $X \rightarrow n X \rightarrow \gamma_{1} n X$ ) is easy. Thus, $k_{1} X=\gamma_{1} n X$ for every $X$.

\subsection{Corollary. $\mathscr{K}_{1}=\mathscr{R}(\mathscr{M} \cap \mathscr{N})$.}

Proof. First note that $\mathscr{R}(\mathscr{M}) \cap \mathscr{R}(\mathscr{N})=\Gamma_{1} \cap \mathscr{N}=\mathscr{K}_{1}$ by 2.1 and 4.1. Now $\mathscr{R}(\mathscr{M} \cap \mathscr{N}) \subset \mathscr{R}(\mathscr{M}) \cap \mathscr{R}(\mathscr{N})$ is obvious. If $X \in \mathscr{R}(\mathscr{M})$, there is a closed embedding $e: X \rightarrow \prod_{\alpha} M_{\alpha}\left(M_{\alpha} \in \mathscr{M}\right)$. Then $e: X \rightarrow \Pi_{\alpha} \pi_{\alpha}(e(X))$ is also a closed embedding, and each $\pi_{\alpha}(e(X)) \in \mathscr{M}$. But for $X \in \mathscr{N}$, each $\pi_{\alpha}(e(X)) \in \mathscr{N} .(\mathscr{N}$ is closed under uniformly continuous images: a discrete set in the image induces one in the domain.) Thus, $\mathscr{R}(\mathscr{M}) \cap \mathscr{N} \subset \mathscr{R}(\mathscr{H} \cap \mathcal{N})$.

4.4. RemarK. For more general $\mathscr{R} \supset \mathscr{K}$, we have this: If $\mathscr{R} \supset \Gamma$, then each $r_{x}$ is an embedding, so $X \subset r_{1} X \subset r X$, by 1.2. If $\mathscr{R} \subset \Gamma \cap \mathscr{N}$, then $\mathscr{K}_{1} \subset \mathscr{R}_{1} \subset \Gamma_{1} \cap \mathscr{N}=\mathscr{K}_{1}$; that is, $\mathscr{R}_{1}=\mathscr{K}_{1}$. Thus, knowledge of $\mathscr{R}_{1}$ (and $r_{1}$ ) is complete except when $\Gamma \notin \mathscr{R} \oplus \Gamma \cap \mathscr{N}$. 


\section{Metric-fine spaces}

We shall discuss briefly the theory of these spaces, and what it has to do with $\mathscr{K}_{1}$ and $\Gamma_{1}$.

A space $X$ is called metric-fine if each uniformly continuous map of $X$ to any metric space $M$ remains uniformly continuous when $M$ is re-equipped with its finest compatible uniformity. These spaces were introduced in Hager (1971), and systematically examined in Hager (1974), Frolík (1974), Rice (1975a), which see.

The property is coreflective in Unif; the metric-fine coreflection of $X$ is denoted $m X$. The space $m e X$ ( $e X$ having base of countable uniform covers of $X$ ) has base of all countable $\operatorname{coz} X$-covers, and $m X$ has base of all $\operatorname{coz} X$-covers which are $\sigma$-discrete with respect to $X$. It follows that $\mathscr{Z}(m X)=\mathscr{Z}($ me $X)=\mathscr{Z}($ e $X)=\mathscr{Z}(X)$ and so the $z$-ultrafilters of $X, e X, m e X$ and $m X$ are all the same. (The space of all $z$-ultrafilters of $X$ is $k m X$, by the way (Hager (1974).)

It follows relatively easily that for a $z$-ultrafilter $\mathscr{F}$ on $X$ (a) $\mathscr{F}$ has cIP if and only if $\mathscr{F}$ is Cauchy for $m e X,(b) \mathscr{F}$ has $\operatorname{co}(\sigma$-disc) IP if and only if $\mathscr{F}$ is Cauchy for $m X$. ((a) is implicit in 8.4 of Hager (1974), (b) is closely related to 3.5 of Rice (1975b)-using (2) of 3.1.) Thus, from 3.2 here, (a) $X \in \mathscr{K}_{1}$ if and only if $m e X \in \Gamma$ (which is almost in 8.1 of Hager (1974)), (b) $X \in \Gamma_{1}$ if and only if $m X \in \Gamma$ (in Rice (1975b)).

That $\mathscr{K}_{1}=\Gamma_{1} \cap \mathscr{N}$ can thus be put: $m e X \in \Gamma$ if and only if $m X \in \Gamma$ and $X \in \mathscr{N}$. This is almost 8.1 of Hager (1974), and is in 2.2 and 2.3 of Rice (1975b). (Each of these results uses Katětov-Shirota Theorem in its proof, of course.)

Rice (1975b) also has results related to 2.1(b) and (c).

\section{References}

H. Curzer and A. W. Hager (1976), 'On the topological completion', Proc. Amer. Math. Soc. 56, 365-370.

Z. Frolík (1974), 'A note on metric-fine spaces', Proc. Amer. Math. Soc. 46, 111-119.

L. Gillman and M. Jerison (1960), Rings of continuous functions (Princeton).

A. W. Hager (1969), 'On inverse-closed subalgebras of $C(X)$ ', Proc. London Math. Soc. (3) 19, 233-257.

A. W. Hager (1971), 'An approximation technique for real-valued functions', General Topology and Applic. 1, 127-134.

A. W. Hager (1974), 'Some nearly fine uniform spaces', Proc. London Math. Soc. (3) 28, 517-546.

A. W. Hager (1975), 'Perfect maps and epi-reflective hulls', Canad. J. Math., 23, 11-24.

J. R. Isbell (1964), Uniform spaces (Providence).

J. Kennison (1965), 'Reflective functors in general topology and elsewhere', Trans. Amer. Math. Soc. 119, 303-315.

M. D. Rice (1975a), 'Metric-fine uniform spaces', J. London Math. Soc. (2) 11, 53-64. 
M. D. Rice (1975b), 'Subcategories of uniform spaces', Trans. Amer. Math. Soc. 201, 305-314.

A. H. Stone (1948), 'Paracompactness and product spaces, Bull. Amer. Math. Soc. 54, 977-982.

Department of Philosophy

University of Texas

Austin, Texas 78712

U.S.A.
Department of Mathematics

Wesleyan University

Middletown, Conn. 06457

U.S.A. 